\title{
EL DILEMA DE LA INFORMALIDAD Y COMO COMBATIRLA
}

\author{
The dilemma of informality and how to combat it
}

Fausto Danilo Erazo Guijarro ${ }^{1}$

Damián Emilio Gibaja Romero²

\section{Resumen}

La informalidad es uno de los principales obstáculos para el desarrollo económico de los países, pues disminuye su productividad. A pesar de esto, la informalidad representa una opción atractiva para cualquier agente productivo dados los beneficios inmediatos que genera. Por ejemplo, mayores ingresos por evasión de impuestos. Sin embargo, el impacto que tiene la informalidad en toda la economía es negativo. El presente estudio analiza la interacción entre gobierno y empresas a partir del dilema del prisionero, en el que se observa que ser informal es una estrategia de equilibrio. Dicho juego nos permite establecer diferentes opciones de política pública para combatir la informalidad. Particularmente analizamos el impacto que la imposición de subsidios y la generación de externalidades tienen en la formalización de agentes productivos y en el gobierno.

\section{Palabras clave}

Economía Informal, Dilema del Prisionero, Equilibrio de Nash, Diseño de Políticas Públicas.

\section{Abstract}

Informality is one of the main obstacles to the economic development of countries, as their productivity decreases. Despite this, informality represents an attractive option for any productive agent due to the immediate benefits it generates; for example, higher income due to tax evasion. However, the impact of informality on the entire economy is negative. The present study analyzes the interaction between government and companies based on the prisoner's dilemma, in which it is observed that being informal is an equilibrium strategy. This game allows us to establish different public policy options to combat informality. We particularly analyze the impact that the imposition of subsidies and the generation of externalities have on the formalization of productive agents and on government.

\section{Key words}

Informal Economy, Prisoner's Dilemma, Nash Equilibrium, Public Policy Design

\footnotetext{
${ }^{1}$ Universidad Nacional de Chimborazo (Ecuador). Correo electrónico: fausto.erazo@unach.edu.ec.

${ }^{2}$ Universidad Popular Autónoma del Estado de Puebla (México). Correo electrónico: damianemilio.gibaja@upaep.mx.
} 


\section{Introducción}

La economía informal incluye todas las actividades productivas, desarrolladas por personas o empresas, que no son declaradas ante las autoridades y que escapan a los registros tributarios y al sistema regulatorio de las mismas (Gómez, 2007). Es decir, un agente productivo es informal cuando no cumple con las obligaciones (impuestos) derivados de la realización de su actividad. En años recientes, el estudio de la economía informal se ha incrementado debido al impacto negativo que tiene en el crecimiento económico de los países. Por definición, el aumento en las actividades informales genera una menor recaudación fiscal, lo que implica que el gobierno tiene menos recursos para garantizar la provisión eficiente de servicios públicos (calidad), a la sociedad o al sector empresarial (Pisani, 2015). También se indica que la informalidad reduce la productividad de un país, debido a que genera una competencia injusta entre agentes formales e informales.

Por lo anterior, la informalidad es una barrera para el desarrollo económico de los países donde el tamaño de dicho sector equipara (o supera) el de las actividades formales (Azevedo \& Almeida, 2009). Así, se observa que los países en desarrollo se caracterizan por poseer un elevado índice de informalidad. En el caso de América Latina y el Caribe, se estima que el sector informal equivale a un $41.1 \%^{1}$ del PIB de la región (Schneider, 2012); mientras que en África, alrededor del $60 \%$ de los empleos son informales (Medina, Jonelis, y Cangul, 2017).

Debido a la magnitud del problema y a sus implicaciones futuras, es necesario que los gobiernos (principalmente de los países en desarrollo) diseñen mejores políticas para disminuir losíndices de informalidad. En el diseño de estas, se debe tener en cuenta que la informalidad representa una opción atractiva para los agentes productivos, pues implica reducir sus costos, ya que no pagan los impuestos correspondientes. En otras palabras, se observa que hay una interacción estratégica entre gobiernos y agentes productivos, en el que el primero busca la obtención de ingresos mediante el pago de impuestos, mientras que los segundos tienen incentivos para no pagarlos pues de este modo, sus beneficios se incrementan.

En el presente artículo se aborda el diseño de políticas públicas de combate a la informalidad. Aunque la informalidad también se relaciona con las fallas de

Como idea ilustrativa, y al no contar con información actualizada, el promedio para el período 1999-2007, el tamaño de la economía informal con relación al PIB estimados por Schneider, en países como Suiza, Estados Unidos, China, Alemania, España e Italia fue de $8,5 \% ; 8,6 \% ; 12,7 \% ; 16 \% ; 22,5 \%$ y $27 \%$ respectivamente. gobierno, llegando a ser esencial para subsistir en países con instituciones políticas y gubernamentales débiles (Eldelbacher, Dobovsek y Kratcoski, 2016), nuestro trabajo se enfoca en analizar los incentivos que un gobierno puede proporcionar para garantizar el registro formal de los agentes productivos. Por consiguiente, analizamos la interacción entre el gobierno y los agentes productivos como un juego simultáneo en el que los jugadores enfrentan el dilema de cumplir con sus responsabilidades u obtener los beneficios de no hacerlo. Así, la interacción es similar al "dilema del prisionero", y a partir de esta observación, estudiamos las consecuencias de la informalidad y aquellas medidas que pueden contribuir a mitigarla. Dichas estrategias se relacionan con generar un comportamiento cooperativo en los jugadores para alcanzar un mayor beneficio social.

El documento se encuentra organizado en seis secciones. En la segunda sección realizamos una revisión de la literatura sobre la informalidad y sus implicaciones. En la tercera sección se describe la interacción entre gobierno y agentes productivos a partir del dilema del prisionero. La siguiente sección estudia el equilibrio del juego presentado en la sección 3, y se discuten las implicaciones sociales de dicha solución. En la sección cinco se presentan diferentes alternativas para modificar el resultado del juego, es decir, estrategias de política pública que incentiven la formalización de los agentes productivos. La última sección presenta las conclusiones.

\section{Revisión de la Literatura}

El primer obstáculo para analizar la informalidad surge de intentar establecer una definición concreta para dicho fenómeno. En la literatura podemos observar que es un concepto cuya conceptualización se puede realizar desde diferentes disciplinas (economía, sociología, derecho, entre otras), y el cual ha cambiado con el paso del tiempo.

El primer intento por establecer una definición precisa de informalidad data de 1972, en estudios realizados por la Organización Internacional del Trabajo (OIT) en Kenia y África. A partir de entonces, se considera al sector informal como toda actividad que no cumple con el pago de impuestos y no se somete a la normatividad. Hart (1972), Mazumdar (1975) y Weeks (1975) mencionan que las actividades informales son actividades que sólo unos pocos están dispuestos a realizar para subsistir. Es decir, estos primeros estudios consideran que la pobreza y la marginalidad son características esenciales de un agente informal. Posteriormente, Tokman (1989) relaciona la informalidad con aspectos microeconómicos 
de la actividad productiva. Menciona que las actividades informales son poco productivas, y los agentes que las realizan son incapaces de acumular capital, ya sea humano o físico. Desde una perspectiva macroeconómica, Loayza (1996) indica que el sector informal se relaciona en gran medida con la evasión de impuestos. Flodman (2004) menciona que la economía informal es la porción no regulada de la economía de mercado que produce bienes y servicios; mientras que Mejía (2007) amplia dicha conceptualización indicando que la informalidad es un conjunto de actividades productivas que no acatan las regulaciones en materias económicas, sanitaria o ambientales, aunque tengan por ley ceñirse a ellas.

En el año 2013, la OIT establece que las empresas informales son aquellas unidades dedicadas a la producción de bienes o a la prestación de servicios, que se caracterizan por funcionar en pequeña escala, con organizaciones rudimentarias, en la que existe muy poca especialización de trabajo y escaso capital como factores de producción que no cumplen con la reglamentación correspondiente a sus actividades.

A partir de las características anteriormente identificadas, es posible establecer una clasificación de las actividades informales en tres grupos (Flórez, 2015). El primer grupo analiza el sector informal como un segmento del mercado laboral. El segundo grupo (que nos compete) considera la informalidad como una actividad ilegal en la que los agentes productivos incurren cuando no pagan los impuestos correspondientes al desarrollo de sus actividades. Finalmente, el tercer grupo observa al mercado laboral informal desde la perspectiva de los trabajadores.

Dada la complejidad de la informalidad, su impacto se refleja no sólo en la productividad del país (Tokman, 1996). Ramos (2015) concluye que las actividades informales son un factor determinante en la generación de desigualdad por ingresos en la población, pues el optar por una actividad informal genera beneficios adicionales en el corto plazo, pero impide que los trabajadores gocen de estabilidad económica en el largo plazo. Por lo anterior, es importante establecer medidas para fomentar la formalización de aquellas empresas que operan fuera de ella. Se sabe que el Programa de Promoción de la Formalización en América Latina y el Caribe, de la Organización Internacional del Trabajo, ha implementado políticas que permiten combatir el sector informal, como reformas tributarias, o la promoción de inversiones productivas.

Nuestro estudio se encuentra estrechamente relacionado con la literatura que analiza la formalización de empresas. Dado que partimos de una interacción estratégica entre gobierno y empresas, el incentivo a la formalización de actividades productivas requiere disminuir el atractivo que genera la evasión de impuestos. Así, la Unión Europea (The World Bank, 2011) fundamenta sus políticas de combate a la informalidad en 4 pilares: acciones preventivas ${ }^{2}$, sanciones ${ }^{3}$, cooperación entre los estados miembros y campañas de sensibilización social.

Mientras que la Comisión Europea y la Organización para la Cooperación y el Desarrollo Económico (OECD, 2015), al igual que la UE, plantean varias políticas que permiten combatir la informalidad. Es así que países como Italia y la ley 383/2001 (Calò, 2001), han aplicado la reducción de impuestos en la oferta. Este es un ejemplo de exenciones fiscales dirigido de manera directa a emprendedores formales. En latinoamérica tenemos el caso de Brasil (Cardoso, 2016), donde se creo el Programa Nacional Simple, que se enfoca de igual forma que Italia a la reducción de impuestos y la simplificación de procedimientos burocráticos para micro, pequeñas y medianas empresas. Es así que la economía informal se presenta como un problema que ha demostrado que es permanente, tanto en paises en desarrollo como en economías avanzadas. Por tal razón, nuestro estudio presenta alternativas de política pública, que permitan combatir la informalidad.

\section{El modelo}

En el presente artículo analizamos la interacción entre gobierno y empresas con el fin de determinar los incentivos necesarios que fomenten la formalización de las primeras. Nótese que esta interacción resume un conflicto en el que ambos agentes siguen un comportamiento estratégico relacionado con la recaudación eficiente de impuestos, por parte del gobierno, y el menor pago de impuestos, por parte de las empresas. A continuación, explicamos dicha interacción en términos del dilema del prisionero.

Sea $S=\{g, a\}$ el conjunto de jugadores, donde $g$ denota al gobierno, y a es un agente productivo (empresa, profesional independiente) representativo. Es decir, asumimos que cada agente productivo interactúa con el gobierno independientemente de las decisiones que otros agentes productivos elijan, pues $g$ proporciona servicios públicos que a utiliza de manera directa para la producción de sus bienes y/o servicios, por ejemplo, infraestructura pública de transporte y/o energía.

\footnotetext{
2 El objetivo es simplificar los procedimientos y las restricciones que limitan la creación de empresas, en particular de nuevas empresas, lo que permitirá eliminar los desincentivos para formalizar la actividad económica.

${ }^{3}$ Parte del fortalecimiento de la vigilancia y la aplicación de sanciones a las personas que se benefician del trabajo clandestino y la protección de las víctimas. De manera particular, a través de una coordinación entre agentes y autoridades.
} 
Entonces, suponemos que $g$ puede elegir entre proporcionar servicios públicos de calidad o no hacerlo; en el primer caso decimos que el gobierno coopera, mientras que la segunda situación refiere lo contrario. Dichas acciones se representan con $C$ y $N C$, respectivamente. De manera similar, asumimos que a decide entre cooperar y no hacerlo. Cuando el agente productivo coopera, esto significa que registra sus actividades con el gobierno, es decir, se formaliza. Así, la no cooperación implica que a decide ser un agente informal.

De lo anterior, se tiene que el conjunto de acciones para $g$ y a es el siguiente $A_{g}=A_{a}=\{C, N C\}$. Es importante resaltar que, aunque en apariencia son el mismo, la interpretación que se da a cada acción es diferente según sea el agente que la elija. También, asumimos que la interacción entre gobierno y agente productivo es simultánea, es decir, los dos eligen al mismo tiempo. En el caso de la formalización, la simultaneidad es un supuesto adecuado debido a que el gobierno no espera a los agentes productivos para proporcionar servicios públicos ya que la sociedad también necesita de ellos. Por su parte, el agente productivo no está consciente de la calidad de los servicios públicos hasta que desarrolla su actividad.

Sabemos que el proporcionar servicios de calidad impacta en el desarrollo de las actividades productivas de $a$, mientras que el pago de impuestos impacta en la capacidad de $g$ para proporcionar servicios públicos de calidad. Es decir, la acción que cada jugador elija impacta en el beneficio que obtiene el otro jugador. Entonces, para tomar una decisión, los jugadores comparan perfiles de acciones. Un perfil de acción, o escenario, es una pareja $(x, y) \in A_{g} \times A_{a}$. Notemos que en la interacción que estamos analizando existen cuatro posibles escenarios. A continuación, describimos el significado de cada uno de ellos.

- Cooperación mutua $(C, C)$. En este escenario ambos jugadores cumplen con sus obligaciones respectivas: el gobierno proporciona servicios de calidad y el agente productivo pagas los impuestos correspondientes a su actividad.

- No cooperación mutua (NC,NC). Esta situación se presenta cuando ninguno de los agentes cumple con sus obligaciones. Así, este caso se refiere a aquella situación en la que el gobierno no proporciona servicios de calidad y el agente productivo desarrolla sus actividades en la informalidad.

- Gobierno coopera y el agente productivo no coopera $(C, N C)$. En este escenario, el agente productivo tiene acceso a servicios públicos de calidad aún cuando no paga el impuesto correspondiente a las actividades que realiza.

- Gobierno no coopera y el agente productivo coopera $(N C, C)$. Situación que se presenta cuando el gobierno no proporciona servicios públicos de calidad a pesar de que el agente productivo se encuentra operando en la formalidad mediante el pago de impuestos.

\section{Pagos de los agentes}

Sabemos que los servicios públicos tienen un impacto positivo en el agente productivo pues le proporcionan acceso a infraestructura pública que le ayuda a desarrollar sus procesos de producción de la mejor manera posible. También, la formalización de las actividades productivas es benéfica para el gobierno pues a partir de ella obtiene los recursos necesarios para generar servicios públicos de calidad, como infraestructura de transporte. En otras palabras, la cooperación (o no) por parte de ambos agentes es crucial para generar (o no) desarrollo económico, y esto se ve reflejado en el beneficio que cada jugador obtiene. Antes de describir los pagos de cada jugador procedemos a introducir notación que nos ayudará a lograr lo anterior.

Sabemos que el pago de cada jugador depende del perfil de acciones, por lo que los beneficios se definen sobre estos. Así, las preferencias de cada jugador están representadas por una función de utilidad $\mathrm{u}_{\mathrm{i}} \operatorname{de} A_{g} \times A_{a}$ en $\boldsymbol{R}$, para todo $i \in J$. En otras palabras, la función de utilidad convierte los perfiles de acciones en un pago.

Notemos que cooperación implica que cada jugador cumpla con sus obligaciones, lo cual implica costos; por lo que no cooperar implica no incurrir en el costo anterior. Es decir, tal como ocurre en el "dilema del prisionero", no cooperar es más atractivo que hacerlo, y esto se obtiene asumiendo la siguiente estructura de preferencias:

$$
u_{i}(N C, C)>u_{i}(C, C) \text { y } u_{i}(N C, N C)>u_{i}(C, N C)
$$

Para todo jugador $i \in J$. Ahora, procedemos a desarrollar el pago/utilidad que cada jugador recibe ante cualquier escenario.

Primero describimos el pago que obtiene $g$. Sabemos que el gobierno obtiene un ingreso positivo I por parte del agente productivo, cuando este coopera. También, se sabe que el gobierno incurre en un costo $C_{1}>0$ cuando ofrece servicios públicos de calidad. Así, cuando el gobierno no genera servicios suponemos que no incurre en dicho costo. Por consiguiente, se tiene que: 


$$
u_{g}(C, C)-I C_{1} \text { y } u_{g}(N C, C)-I
$$

Cuando el agente productivo elige no cooperar, el gobierno no recibe el ingreso $I$ que obtendría con el pago de impuestos. Así, cuando el gobierno coopera y los agentes productivos no, $g$ obtiene un pago - $C_{1}$, en otras palabras, el gobierno pierde como consecuencia de no recibir impuestos. Por otra parte, cuando el gobierno no coopera, no ofrece servicios públicos de calidad (no incurre en costos), y al no tener ingresos provenientes de impuestos, el pago que obtiene es cero. Por consiguiente, los pagos que el gobierno obtiene cuando el agente productivo no coopera son los siguientes:

$$
u_{g}(C, N C)=-C_{1} \text { y } u_{g}(N C, N C)=0
$$

Ahora, vamos a describir el pago del agente productivo. En el caso de que el gobierno coopere, el agente productivo tiene acceso a servicios de calidad que le ayudan a su actividad productiva. El contar con servicios de calidad le proporciona al agente productivo una utilidad $U(S C)>0$, la cual resume el valor de llevar a cabo eficientemente sus procesos de producción. En el caso de que a coopere, esto implica que paga una cantidad de impuestos I por formalizar su actividad, o no los paga en caso de que elija no cooperar. Entonces, los pagos que recibe el agente productivo cuando el gobierno coopera son los siguientes:

$$
u_{a}(C, C)=U(S C)-I \text { y } u_{a}(N C, C)=U(S C)-I
$$

Cuando el gobierno no coopera, el agente productivo tiene acceso a un servicio público de mala calidad, que impacta negativamente en sus actividades productivas. Dicho impacto se traduce en un ingreso $U(M C)<U(S C)$. Así, los pagos que el agente productivo obtiene cuando el gobierno no coopera son los siguientes:

$$
u_{a}(C, N C)=U(M C)-I \text { y } u_{a}(N C, N C)=U(M C) .
$$

La Tabla 1 resume la discusión anterior, por lo que muestra la matriz de pagos del juego.

Tabla 1. Dilema del prisionero, matriz de pagos

\begin{tabular}{|l|l|c|c|}
\hline \multicolumn{2}{|c|}{} & \multicolumn{2}{|c|}{ Agentes Productivos (a) } \\
\cline { 3 - 4 } \multicolumn{2}{|c|}{} & C & NC \\
\hline \multirow{2}{*}{ Gobierno (g) } & C & $I-C 1 ; U(S C)-1$ & $-\mathrm{C} 1 ; \mathrm{U}(\mathrm{SC})$ \\
\cline { 2 - 4 } & $\mathrm{NC}$ & $I ; U(M C)-1$ & $0 ; \mathrm{U}(\mathrm{MC})$ \\
\hline
\end{tabular}

Fuente: elaboración propia.

\section{Análisis de Equilibrio}

En la Tabla 1 podemos observar que la no cooperación induce el mayor pago posible para cada jugador, sin importar la acción que elija el otro. A continuación, analizamos las implicaciones que esto tiene en la solución el juego.

\section{El equilibrio de Nash en el juego de informalidad}

La solución de un juego puede ser definida desde diferentes perspectivas. Por ello, su resolución es diferente a la de los problemas de optimización clásica. Por ejemplo, podemos resolver un juego ya sea en términos de dominancia o de equilibrio. El primer enfoque busca que los agentes obtengan siempre la mejor solución sin importar la estrategia que elijan los otros jugadores, mientras que el segundo enfoque pretende encontrar un perfil de estrategias bajo el que cual los jugadores no tengan incentivos a cambiar sus acciones, perfil que también se conoce como equilibrio de Nash.

El equilibrio de Nash es una solución debido a que, a diferencia del concepto de dominancia, siempre es posible encontrar un perfil con sus características sin importar el juego. Por ello se dice que dicho concepto de solución constituye una predicción válida sobre el posible comportamiento de los jugadores.

Decimos que $x_{g}^{*}, x_{a}^{*} \in A_{g} x A_{a}$ es un equilibrio de Nash si:

$u_{g}\left(x_{g}^{*}, x_{d}^{*}\right) \geq u_{g}\left(x_{g}^{*}, x_{d}^{*}\right)$ y $u_{a}\left(x_{g}^{*}, x_{d}^{*}\right) u_{a}\left(x_{g}^{*}, x_{d}^{*}\right)$

Para todo $x_{g} \in A_{g}$ y $x_{a} \in A_{a}$. En otras palabras, $x_{g}^{*}$ es una estrategia de equilibrio para el gobierno, si maximiza el beneficio que este obtiene cuando el agente productivo elige $x_{a}^{*}$. La misma interpretación se puede obtener de manera análoga para el agente productivo.

La observación anterior nos permite obtener un proceso para la búsqueda del equilibrio de Nash: dada una acción elegida por el agente productivo, el gobierno debe buscar la acción que le proporcione el mayor pago posible. Lo mismo debe hacer el agente productivo ante cada posible acción que elija el gobierno. El proceso anterior se conoce como la búsqueda de la mejor respuesta. Entonces, las estrategias de equilibrio son la mejor respuesta ante lo que el otro jugador seleccione como estrategia de equilibrio.

El proceso anterior se ejemplifica con los cuatro escenarios 
que se resumen en la tabla 1. Nótese que, sin importar si el agente productivo se vuelve formal o informar, el gobierno siempre obtiene un mayor beneficio cuando no proporciona un servicio de calidad. En otras palabras, la mejor respuesta del gobierno ante cualquier acción del agente productivo es no cooperar. Por otra parte, sabemos que la formalización del agente productivo implica pagar un impuesto, mientras que no hacerlo implica no incurrir en dicho costo. Sin embargo, aunque no sea un agente formal, el gobierno le sigue proporcionando servicios públicos, es decir, no cooperar es la mejor respuesta ante cualquier acción que elija el gobierno.

De la discusión anterior se observa que la mejor respuesta del gobierno a la informalidad del agente productivo es proporcionar servicios de mala calidad, y la mejor respuesta del agente productivo a los servicios de mala calidad es desarrollarse en la informalidad. Lo anterior se cumple cuando iniciamos con el agente productivo. Por lo tanto, no cooperar es la mejor respuesta para cualquier jugador, de lo que se concluye que $(N C, N C)$ es el único equilibrio de Nash del juego; es decir, el gobierno y el agente productivo no tienen incentivos a cambiar su comportamiento no cooperativo. Además, dicho equilibrio también es dominante, pues no cooperar siempre proporciona el mayor pago posible a cada jugador sin importar la acción que el otro jugador elija. La Tabla 2 resume la discusión anterior. En azul se resalta el pago que cada jugador recibe en el perfil de equilibrio.

Tabla 2. Pagos en el equilibrio de Nash

\begin{tabular}{|c|c|c|c|}
\hline & & \multicolumn{2}{|c|}{ Agentes Productivos (a) } \\
\hline & & C & $\mathrm{NC}$ \\
\hline \multirow{2}{*}{ Gobierno (g) } & $\mathrm{C}$ & $I-C_{1} ; U(S C)-1$ & $-\mathrm{C}_{1} ; \mathrm{U}(\mathrm{SC})$ \\
\hline & $\mathrm{NC}$ & $I ; U(M C)-1$ & $0 ; \mathrm{U}(\mathrm{MC})$ \\
\hline
\end{tabular}

Fuente: elaboración propia.

\section{Implicaciones del equilibrio de Nash}

Cuando definimos el equilibrio de Nash, mostramos que su búsqueda es equivalente a encontrar la mejor respuesta de cada jugador ante cada acción del otro jugador; y el equilibrio se constituye cuando la acción es la mejor respuesta a la mejor respuesta del otro jugador. Este proceso indica una búsqueda unilateral de la solución, lo cual se traduce en un comportamiento egoísta que explicaremos a continuación. Primero, debemos recordar lo que cada escenario significa:
Tabla 3. Significado de cada posible escenario en la formalización del agente productivo

\begin{tabular}{|c|c|c|}
\hline Acciones $(\boldsymbol{g})$ & Acciones $(\boldsymbol{a})$ & Ambos \\
\hline$C$ & $C$ & $\begin{array}{c}\text { Obras de calidad } \\
\text { Pago de impuestos }\end{array}$ \\
\hline$C$ & $N C$ & $\begin{array}{c}\text { Obras de calidad } \\
\text { Evasión de impuestos }\end{array}$ \\
\hline$N C$ & $C$ & $\begin{array}{c}\text { Obras de mala calidad } \\
\text { Pago de impuestos }\end{array}$ \\
\hline$N C$ & $N C$ & $\begin{array}{c}\text { Obras de mala calidad } \\
\text { Evasión de impuestos }\end{array}$ \\
\hline
\end{tabular}

Fuente: elaboración propia.

Nótese que el equilibrio corresponde al resultado donde el agente productivo evade impuestos y el gobierno no genera servicios de calidad. Dicho equilibrio emerge pues del no cumplimiento de las obligaciones correspondientes. Implica no incurrir en sus costos asociados. Sin embargo, como podemos observar en la Tabla 2, los pagos que se obtienen cuando ambos jugadores cooperan son mayores a los que se obtienen en equilibrio. En otras palabras, aunque la informalidad genera beneficios individuales, socialmente no es la mejor alternativa pues se encuentran peor que en la situación en la que ambos cooperan.

Lo anterior nos permite las siguientes observaciones:

- La decisión egoísta nos lleva a un escenario donde el bien común no se alcanza. Es decir, el gobierno no puede proporcionar servicios de calidad por la falta de recursos, mientras que el agente productivo no explota todas sus capacidades por la ausencia de servicios públicos de calidad.

- La decisión del bien común no es un equilibrio a pesar de que es la mejor en términos sociales. Es decir, es necesario diseñar mecanismos para alcanzar el bien común debido a que por sí sólo genera incentivos para que gobierno y el agente productivo cambien su comportamiento.

Por lo tanto, el equilibrio de Nash representa una solución no cooperativa y subóptima en sentido paretiano ${ }^{4}$ (García López \& Pérez Forniés, 2001).

\section{Incentivos para cooperar}

La sección anterior muestra la necesidad de una política pública que incentive la formalización de los agentes

\footnotetext{
${ }^{4}$ El economista Italiano Vilfredo Pareto (1848-1923) desarrolló el concepto de óptimo, y este detalla la situación en la que no es posible beneficiar a un agente sin perjudicar a otro. En su búsqueda de llegar a un equilibrio y alcanzar un bienestar, nuestros agentes aceptan ceder hasta el punto en el que deja de generar beneficios. El óptimo de Pareto se fundamenta en criterios de utilidad, es decir si algo produce un bienestar común despertará un proceso natural de optimización y permitirá alcanzar un punto óptimo.
} 
productivos pues la ausencia de esta induce un equilibrio socialmente subóptimo en el que toda la población se ve afectada pues el gobierno ofrece servicios de mala calidad. La presente sección explora aquellas circunstancias que contribuyen a garantizar tanto la formalización del agente productivo como la provisión de servicios públicos de calidad.

\section{Los subsidios}

El dilema de prisionero indica que el agente productivo tiene incentivos para no formalizarse, pues dicho proceso implica costos, que reducen su beneficio. Por lo anterior, es claro que, sí queremos inducir en el agente productivo un comportamiento colaborativo, la formalización le debe proporcionar pagos superiores al costo que conlleva realizar dicho proceso. En otras palabras, formalizarse debe generar siempre el mayor pago posible sin importar la acción que el gobierno elija, lo que se traduce en el siguiente sistema de desigualdades:

$$
u_{a}(C, C)>u_{a}(C, N C) \text { у } u_{a}(N C, C)>u_{a}(N C, C)
$$

Es decir, el impuesto $I$, relacionado con la formalización de la actividad productiva, debe satisfacer lo siguiente:

$$
U(S C)-I>U(S C) \text { y } U(M C)-I>U(M C)
$$

Al resolver el sistema anterior, en términos de $I$, observamos que dicho impuesto debe ser negativo para garantizar que la cooperación sea una estrategia dominante para el agente productivo. Dicha situación corresponde al caso en que el gobierno le proporciona un subsidio al agente productivo para formalizarse. A pesar de que un subsidio garantiza la formalización del agente productivo, observemos que dicha estrategia es costosa para el gobierno pues el gobierno tiene que cubrir el costo de generar servicios públicos y al mismo tiempo renuncia al ingreso que el proceso de formalización proporciona. Por consiguiente, el gobierno sigue teniendo incentivos para generar servicios de mala calidad pues dicha situación le resulta menos costosa. Por lo tanto, los subsidios generan una solución en la que las empresas se formalizan, pero el gobierno ofrece servicios de mala calidad.

\section{Externalidades}

En los supuestos del modelo establecimos que la provisión de servicios de calidad le genera al agente productivo una utilidad directa, pues este los utiliza para desarrollar sus actividades productivas de manera eficiente. Por ejemplo, la infraestructura de transporte y la provisión de energía tienen un impacto directo en todo agente productivo. Sin embargo, esto no basta para incentivar la formalización de las empresas, como lo indica el dilema del prisionero.

A pesar de lo anterior, existen otros servicios que generan un impacto indirecto en el agente productivo y que este puede percibir que son consecuencia de formalizarse. Garantizar seguridad, así como un entorno financiero sólido y bien regulado, son servicios que generan confianza entre posibles inversionistas que impactan zde manera indirecta, pero positivamente, en los beneficios que obtiene un agente productivo. Por ejemplo, cuando el gobierno proporciona confianza, los inversionistas tienen incentivos para apoyar a las empresas. Asimismo, la promoción de empresas mediante ferias nacionales e internacionales, son servicios que el gobierno ofrece y que tienen un impacto positivo, aunque de manera indirecta, en las ganancias del agente productivo.

Los aspectos mencionados anteriormente, forman parte de las dimensiones de la seguridad que son consideradas para el desarrollo económico (GuhaKhasnobis, Kanbur, \& Ostrom, 2006). Denotamos por $x_{g}$ la externalidad positiva que las empresas obtienen de manera indirecta por parte de servicios públicos que no son utilizados directamente en sus procesos de producción; es importante mencionar que $\boldsymbol{a}$ recibe dicha externalidad cuando registra sus actividades en el gobierno y este coopera, lo cual corresponde al escenario $(C, C)$. Lo anterior se debe a que el sistema financiero y las ferias comerciales se restringen a apoyar a empresas formales ya que es más fácil medir el impacto de este tipo de estrategias. Además, cuando las externalidades que genera el estado son significativas, las empresas son conscientes de ello por lo que se convierte en un costo de oportunidad cuando deciden no formalizarse.

Notemos que la formalización de las empresas, además de registrar sus actividades productivas ante el estado, también implica un mejor monitoreo del gobierno a los trabajadores de estas. Es decir, la regulación del mercado laboral mejora con la formalización de los agentes productivos, y ello a su vez conlleva a un mejor diseño de políticas públicas contra el desempleo, en la oferta de servicios de salud y en el control de los salarios, con efectos positivos en el bienestar de la población (GuhaKhasnobus, Kanbur y Ostrom, 2006). Así, denotamos por $X_{a}$ la externalidad positiva que el registro de las empresas genera en el gobierno. De igual manera, observamos que el gobierno puede acceder a dicha externalidad cuando las 
empresas se registran. Además, notemos que el gobierno percibe dicha externalidad como un costo de oportunidad cuando no se preocupa por proveer servicios de calidad; dicho de otra manera, el no proporcionar servicios de calidad implica que el gobierno no está usando de manera adecuada sus recursos, por lo que los beneficios que podría obtener de la formalización son desaprovechados.

La tabla 4 resume la discusión anterior. De acuerdo con las externalidades relacionadas con la formalización y la oferta de servicios de calidad, notamos cambios en el pago que cada jugador recibe en cada escenario.

Tabla 4. Impacto de las externalidades en los pagos.

\begin{tabular}{|c|c|c|c|}
\hline & \multicolumn{2}{|c|}{ Agentes Productivos (a) } \\
\hline & & C & - $\mathrm{NC}$ \\
\hline \multirow{2}{*}{ Gobierno $(g)$} & C & $I-C_{1}+x_{a} ; U(S C)+x_{g}-I$ & - C1; U(SC) \\
\hline & NC & $I-x_{a} ; U(M C)-I$ & $0 ; U(M C)-x_{g}$ \\
\hline
\end{tabular}

Fuente: elaboración propia.

De la tabla 4, podemos concluir que el escenario $(C, C)$ es un equilibrio de Nash cuando se cumplen las siguientes condiciones:

$$
x_{g}>I \text { y } x_{a}>C_{1}
$$

En otras palabras, la empresa debe recibir un beneficio mayor que el pago que hace por registrarse, mientras que el bienestar que se logra con la población debe ser mayor que el costo de ofertar servicios de calidad.

\section{Conclusiones}

El presente artículo establece que la informalidad es el resultado de un dilema social, en el que el agente productivo y el gobierno buscan de manera unilateral maximizar su beneficio. Dicho análisis, basado en la interacción entre ambos agentes, permite establecer posibles soluciones de política pública. La primera solución, subsidios al proceso de formalización, resulta costoso para el gobierno y lo lleva a formalizar a todas las empresas sacrificando la calidad de los servicios.

Sin embargo, es posible generar un comportamiento cooperativo por parte del gobierno repitiendo el razonamiento que se siguió para el establecimiento de los subsidios. Dicho análisis da como resultado costos negativos sobre la provisión de servicios públicos, lo cual indica que el gobierno proveerá servicios públicos de calidad cuando la oferta de estos le genere beneficios mayores al costo de producirlos. Es decir, el gobierno tiene ingresos que le permiten subsidiar la formalización del agente productivo y también cubrir los costos asociados a la producción de servicios públicos. El problema central de este escenario radica en que la obtención de beneficios se relaciona con la recaudación fiscal y las actividades del Estado, aspectos que se encuentran fuera de nuestro modelo. Sin embargo, es un aspecto que pretendemos abordar en el futuro.

Finalmente, una opción menos costosa es enfocarse en las externalidades que tanto el Estado como las empresas pueden generar. Lo anterior, se puede lograr a través de un gasto más eficiente por parte del gobierno, que es una alternativa menos costosa a la provisión de subsidios. Esta alternativa relaciona de manera directa el problema de la informalidad con las fallas de gobierno, tal como lo señalan Parry et al. (2007).

\section{Referencias}

1. Azevedo, R., y Almedia, N. (2009). An evolutionary game theory approach to the dynamics of the labour market: A formal and informal perspective. Structural Change and Economic Dynamics, 21(2), 101-110. doi: https://doi.org/10.1016/j. strueco.2009.11.002

2. Banco Mundial. (2008). Informe sobre crecimiento. Estrategias para el crecimiento sostenido y el desarrollo incluyente. Comisión para el crecimiento y el desarrollo. Colombia: Mayol Ediciones S.A.

3. Bustillo, I., y Velloso, H. (2017). Insecurity and Development in Latin Ametica and the Caribbean. PRISM: A Journal of the Center for Complex Operations, 5(4), 48-67.

4. Cardoso, A. (2016). Informality and public policies to overcome it. The case of Brazil. Sociologia \& Antropologia, 6(2), 321-349. doi: http://dx.doi. org/10.1590/2238-38752016v622

5. Flodman, B. K. (2004). The informal economy: Fact finding study. Stockholm Sweden: ORGUT Consulting AB.

6. Flórez, L. A. (2015). The Search and Matching Equilibrium in an Economy with an Informal Sector: A Positive Analysis of Labour Market Policies. Revista Desarrollo y Sociedad, 75, 51-99. doi: https:// 
doi.org/10.13043/dys.75.2

7. Forniés, C. P., y López, J. M. G. (2001). Equilibrios de Nash y de Cournot: El caso de la provisión privada de un bien público. Acciones e investigaciones sociales, $13,75-85$.

8. Gómez Naranjo, L. G. (2007). La informalidad en la economía, algo incuestionable. Semestre Económico, 10(19), 47-67.

9. Guha-Khasnobis, B., Kanbur, R., \& Ostrom, E. (2006). Beyond formality and informality. En GuhaKhasnobis, B., Kanbur, R., \& Ostrom, E. (Eds), Linking the formal and informal economy. Concepts and policies (pp. 75-92). Oxford University Press.

10. Loayza, N. (1996). The Economics of the Informal Sector: A Simple Model and Some Evidence from Latin America. Carnegie-Rochester Conference Series on Public Policy, 45, 129-162. doi: https://doi. org/10.1016/S0167-2231(96)00021-8.

11. Medina, L., Jonelis, M., \& Cangul, M. (2017). The informal economy in Sub-Saharan Africa: Size and determinants. IMF Working paper WP/17/156. International Monetary fund.

12. Mejía, D. (2007). Informalidad: teoría e implicaciones de política. Serie Borradores de economía No. 455. Banco de la Respública. Bogotá, Colombia.

13. Herrera, J., y Hidalgo, N. (2014). Dynamics of informal microenterprises and poverty in Peru: a panel approach. En Cling, J.P., Lagrée, S., Razafindrakoto, M., y Roubaud, F. (Eds.) The Informal Economy in Developing Countries (pp. 324-340). London: Routledge. doi: https://doi. org/10.4324/9781315850283

14. Naciones Unidas EL SALVADOR. (2014). Economía Informal: ¿Deomocracia a medias? San Salvador: Sistema de las Naciones Unidas en El Salvador.

15. Norman V. Loayza, A. M. (2005). The Impact of Regulation on Growth and informality: Cross Country Evidence. . World Bank Policy Working Paper 3623.

16. Calò. E. (2001). Informe Nacional Italia. Notarius
International, 3-4, 196-211

17. OECD. (2015). Policy Brief on Informal Entrepreneurship. Luxembourg: European Unio/ OECD.

18. OIT (Organización Internacional del Trabajo). (1972). Instrumentos, resolución y otros textos adoptados por la Conferencia Internacional del Trabajo. Ginebra: Boletin Oficial.

19. (2013). La medición de la Informalidad: Manual estadístico sobre el sector informal y el empleo informal. Turin: Centro internacional de Formación de la OIT.

20. Parry, G., Arias, O., Fajnzylber, P., Maloney, W., Mason, A., y Saavedra-Chanduvi, J. (2007). Informality: Exit and exclusion. The International Bank for Reconstruction and Development / The World Bank. doi: https://doi.org/10.1596/978-08213-7092-6

21. Pisani, M. J. (2015). Does Informality Impact Formal Sector Firms?: A Case Study from Nicaragua. The Journal of Developing Areas, 49(2), 317-334. doi: $10.1353 /$ jda.2015.0033

22. Ramos, A. (2015). Informal sector, informal economy and informality. Guadalajara: RIDE.

23. Schneider, F. (2012). The Shadow Economy and Work in the Shadow: What Do We (Not) Know? Germany: IZA DP N 6423,24.

24. The World Bank. (2011). Policies to Reduce Informal Employment an international survery. Ukraine.

25. Tokman, V. (1989). Policies for a Heterogeneous Informal Sector in Latin America. Great Britain: Pergamon Press plc.

26.

(2004). Una voz en el camino: empleo y equidad en América Latina 40 años de búsqueda. Santiago de chile: FCE. 